of stress and an elongation that increased the cellular area. This suggests that intermediate filaments might protect superelastic cells from undergoing an unlimited deformation by acting like springs that resist tension at high levels of deformation. In such circumstances, the ability of intermediate filaments to return to their usual length after being stretched might even enable such cells to recover their initial shape when tension is released.

Latorre and colleagues' work has revealed a more complex relationship between cell size and the forces that cells experience than was previously appreciated. Future studies should attempt to unravel the mechanisms that enable cells to enter a state of superelasticity and to recover from high levels of deformation. Now that we know cell shape is not an appropriate proxy for assessing cellular tension, it will be crucial to develop ways to accurately monitor tension levels in tissues, so as to better understand the factors that influence tissue shape.

Manuel Théry is at UMR 1160, Paris Diderot

University, Saint-Louis Hospital, CEA, INSERM, AP-HP, 75010 Paris, France, and at UMR5168, University Grenoble-Alpes, BIG/LPCV, CEA, CNRS, INRA, Grenoble, France. Atef Asnacios is at UMR7057 CNRS, Paris Diderot University, MSC, 75205 Paris Cedex 13, France.

e-mails: manuel.thery@cea.fr;

atef.asnacios@univ-paris-diderot.fr

1. Kipling, R. Just So Stories (Macmillan, 1902).

2. Latorre, E. et al. Nature 563, 203-208 (2018).

3. Trepat, X. et al. Nature Phys. 5, 426-430 (2009).

4. Fernández, P., Pullarkat, P. A. \& Ott, A. Biophys. J. 90, 3796-3805 (2006).

5. Gardel, M. L. et al. Proc. Natl. Acad. Sci. USA 103 1762-1767 (2006).

6. Fabry, B. et al. Phys. Rev. Lett. 87, 1-4 (2001).
7. Lenormand, G., Millet, E., Fabry, B., Butler, J. P. \& Fredberg, J. J. J. R. Soc. Interface 1, 91-97 (2004).

8. Desprat, N., Richert, A., Simeon, J. \& Asnacios, A. Biophys. J. 88, 2224-2233 (2005).

9. Bonakdar, N. et al. Nature Mater. 15, 1090-1094 (2016).

10.Webster, K. D., Ng, W. P. \& Fletcher, D. Biophys. J. 107, 146-155 (2014).

11. Harris, A. R. et al. Proc. Natl Acad. Sci. USA 109, 16449-16454 (2012).

12. Mitrossilis, D. et al. Proc. Natl Acad. Sci. USA 106, 18243-18248 (2009).

13. Trepat, X et al. Nature 447, 592-595 (2007).

14.Burke, T. A. et al. Curr. Biol. 24, 579-585 (2014)

15.Chugh, P. et al. Nature Cell Biol. 19, 689-697 (2017)

16. Ennomani, H. et al. Curr. Biol. 26, 616-626 (2016).

17. Ouinlan, R. A. et al. J. Cell Sci. 130, 3437-3445 (2017).

18.Mendez, M. G., Restle, D. \& Janmey, P. A. Biophys. J. 107, 314-323 (2014)

19. Kreplak, L., Bär, H., Leterrier, J. F., Herrmann, H. \& Aebi, U. J. Mol. Biol. 354, 569-577 (2005).

20.Troyanovsky, S. M., Eshkind, L. G., Troyanovsky, R. B., Leube, R. E. \& Franke, W. W. Cell 72, 561-574 (1993).

This article was published online on 31 October 2018.

\title{
Immune-cell crosstalk in multiple sclerosis
}

\section{Interactions between the $B$ and $T$ cells of the human immune system are implicated in the brain disease multiple sclerosis. It emerges that B cells make a protein that is also made in the brain, and that $T$ cells recognize this protein.}

\section{RICHARD M. RANSOHOFF}

A hallmark of the disease multiple sclerosis is an inflammatory autoimmune attack ${ }^{1}$ on the proteins of the myelin sheath, a structure that wraps around the nerve fibres that project from neurons. The myelin sheath provides protection and nourishment to nerve fibres and enables efficient transmission of nerve impulses. Myelin-sheath injury causes a range of symptoms, depending on the neurons that are affected. Which immune-system cells and protein targets have key roles in the initiation and progression of multiple sclerosis is not fully understood, and such information might aid the development of new treatments. Writing in Cell, Jelcic et al. ${ }^{2}$ present an analysis of immune-system cells found in people with multiple sclerosis that deepens our understanding of how immune cells might contribute to this disease.

One factor linked ${ }^{3,4}$ to the risk of developing multiple sclerosis is the possession of a particular version of a protein called HLA. HLA proteins enable cells to display antigens - fragments of proteins - on their surfaces. If the receptor for an antigen (the T-cell receptor; TCR) on a $\mathrm{T}$ cell recognizes an antigen presented by an HLA protein, the $\mathrm{T}$ cell is activated to trigger an immune response against cells that express the antigen.
Variations in the antigen-binding capacity of different HLA proteins and in the antigenrecognition capacity of TCRs enable the body to respond to a wide range of antigens associated with disease-causing microorganisms. However, there is a danger that if an HLA protein efficiently binds an antigen that is normally part of the body, and if a $\mathrm{T}$ cell that recognizes the HLA-antigen complex is activated, autoimmunity could develop. Such a mechanism might underlie the fact that the version of HLA called HLA-DR15 is a risk factor for multiple sclerosis ${ }^{3}$, and is estimated ${ }^{4}$ to contribute $60 \%$ of the total genetic risk for developing the condition.

$\mathrm{T}$ cells from people with multiple sclerosis are more prone to divide in vitro than are T cells from people without the condition ${ }^{3}$. Such cell division is reminiscent of the division that occurs as the result of normal immune-cell activation by an antigen stimulus, but in this case it does not seem to require the addition of an antigen stimulus to the sample of immune cells $^{3}$. This suggests either that the normal requirement for antigen recognition is being bypassed, or that these $\mathrm{T}$ cells recognize an antigen that is present on other immune cells in the blood sample. Jelcic et al. investigated further, analysing in more detail the behaviour of immune cells in blood samples of people with multiple sclerosis. They convincingly demonstrate that both $\mathrm{T}$ cells and another type of immune cell called a B cell from these samples could proliferate when grown in vitro. The authors term this type of division autoproliferation, because it occurs spontaneously in vitro without the addition of an antigen.

Jelcic et al. found that signalling through a TCR-initiated T-cell proliferation, and that cell proliferation was associated with the production by $\mathrm{T}$ cells of a signalling protein called IFN- $\gamma$ (Fig. 1), which is associated with multiple sclerosis ${ }^{5}$. IFN- $\gamma$ is a potent activator of a category of immune cells called macrophages, which directly damage the myelin sheath ${ }^{6,7}$ in multiple sclerosis.

The authors implicate B-cell proliferation in driving T-cell autoproliferation, because neither T cells nor B cells divided if the cultured cells were exposed to a drug called ibrutinib. Ibrutinib inhibits the protein BTK, which is essential for signalling downstream of the B-cell antigen receptor that leads to B-cell proliferation ${ }^{8}$. Interestingly, a phase IIb clinical trial (see go.nature.com/2yhfphu) has reported preliminary evidence that the BTK inhibitor evobrutinib could potentially provide benefit for people with multiple sclerosis (see go.nature.com/2qtqby9).

Each of the multiple-sclerosis treatments currently in use suppresses disease-associated brain inflammation, but in different ways. Jelcic et al. took advantage of this to test whether interactions between B cells and $\mathrm{T}$ cells are needed for T-cell autoproliferation, and whether this phenomenon might be involved in processes that lead to the symptoms of multiple sclerosis.

The authors analysed blood samples from people with the disease who were receiving different anti-inflammatory treatments, and compared these results with control samples from people with the disease who were not receiving treatment. For those receiving an antibody called natalizumab, which causes an increase in the numbers of $\mathrm{T}$ cells and 
immature B cells in the blood, in vitro analysis showed that autoproliferation of B cells and $\mathrm{T}$ cells was increased compared with the controls. Samples from those receiving an antibody called rituximab, which depletes B cells from the bloodstream, had much-reduced T-cell proliferation compared with controls.

This analysis of the effect of anti-inflammatory treatments that affect $\mathrm{T}$ cells or $\mathrm{B}$ cells provides evidence consistent with the authors' model that clinically relevant interactions between $\mathrm{B}$ cells and T cells occur in multiple sclerosis. For many years, it was generally thought that B cells do not have a role in multiple sclerosis, because of results from animal studies ${ }^{9}$. This view changed when striking benefits were observed in clinical trials of B-cell depletion for multiple-sclerosis treatment ${ }^{10-12}$.

Jelcic and colleagues needed to answer the question of whether the autoproliferating T cells contribute to the development of multiple sclerosis. To address this tough problem, the authors analysed the cellular descendants of individual proliferating $\mathrm{T}$ cells from the blood of people with multiple sclerosis. They looked at the variable portions of the TCRs present in the cells because these variable regions provide a unique pattern, akin to a barcode, that can identify any $\mathrm{T}$ cell and its genetically identical descendants - which form a cellular lineage termed a clone.

In rigorous and challenging experiments using material from two people who had multiple sclerosis, the authors analysed T cells found in brain tissue taken at biopsy or postmortem and compared these with T cells from the same person's blood samples taken before brain-tissue samples were obtained. The authors found that $\mathrm{T}$ cells from blood samples that underwent autoproliferation in vitro belonged to an identical cellular lineage that matched $\mathrm{T}$ cells found in the brain-tissue samples taken from the same person.

This finding strongly suggests that some proliferating cells in the blood of people with multiple sclerosis could enter their brain. Once there, such T cells might release immunesignalling molecules called cytokines, such as macrophage-stimulating IFN- $\gamma$, that could initiate inflammatory tissue injury. Immune cells are always present in the cerebrospinal fluid that bathes the brain and spinal cord, and IFN- $\gamma$-producing T cells ${ }^{13,14}$ and proliferating $\mathrm{T}$ cells ${ }^{15}$ have previously been identified in the cerebrospinal fluid of individuals with multiple sclerosis. Jelcic and colleagues' findings therefore highlight the significance of previous observations and provide additional support for established models of how this disease proceeds.

A final conundrum remains: what are the antigenic target(s) of these T cells? This is a key question because the relevant selfantigens driving multiple sclerosis have not been definitively identified. To try to answer this, the authors analysed a clonal cell population grown in vitro, derived from one T cell

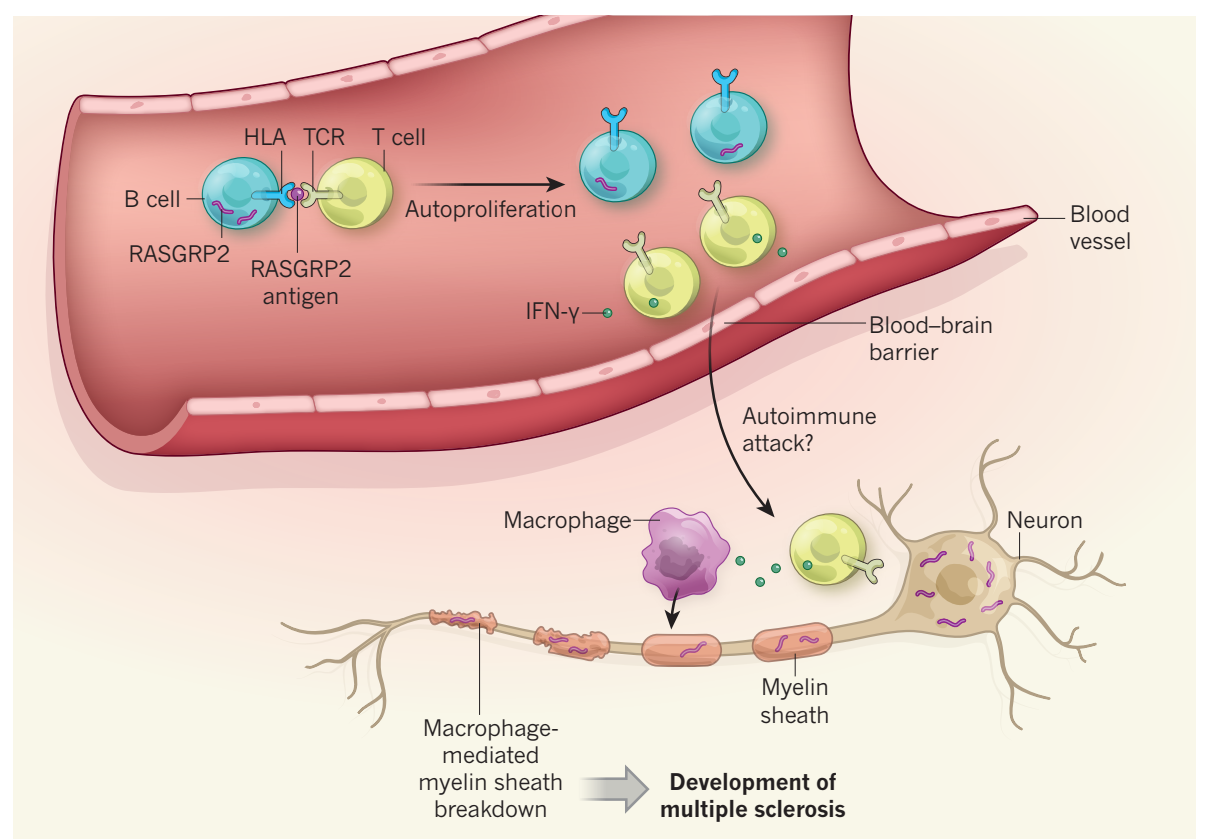

Figure 1 | Immune-cell action associated with multiple sclerosis. Jelcic et al. ${ }^{2}$ report that B cells of the immune system present in the bloodstream make a protein called RASGRP2. These cells use a protein called HLA to present a peptide fragment (an antigen) of RASGRP2 on their cell surface. If this antigen is recognized by the T-cell receptor (TCR) of another immune cell called a T cell, this interaction leads to the proliferation of both the T cells and the B cells, a phenomenon that the authors call autoproliferation. Their evidence indicates that these autoproliferating T cells can, by an unknown route, cross the bloodbrain barrier to enter the brain. RASGRP2 is also found in brain tissue. If neurons or other brain cells express RASGRP2, this might trigger T cells that infiltrate the brain to orchestrate an autoimmune attack by producing inflammatory mediators. For example, the production of IFN- $\gamma$ proteins by activated T cells could stimulate the macrophages of the immune system, which are known ${ }^{6,7}$ to attack the myelinsheath structure that protects nerve fibres and supports neuronal function. This in turn could lead to the development of multiple sclerosis.

and its descendants that were present in the blood and the post-mortem brain of a person with multiple sclerosis. Jelcic and colleagues used an innovative approach that relied on computational evaluation of data obtained using standard methods to stimulate T cells. The authors evaluated an almost unimaginably large number of structurally similar but nonidentical peptides for their capacity to act as an antigen that would generate a response from this T-cell population. They uncovered an antigen from the protein RASGRP2 as one that probably stimulates the TCR of this T-cell population. RASGRP2 had not previously been linked to processes related to multiple sclerosis. The authors demonstrated that RASGRP2 is expressed both in B cells that elicit $\mathrm{T}$-cell proliferation and in brain tissue.

Jelcic and colleagues' study provides a model for how B-cell and T-cell interactions outside the brain might generate diseasecontributing $\mathrm{T}$ cells that then enter the brain. Their discovery of an antigen associated with multiple sclerosis might, if other such antigens are identified in the future, reveal how autoimmunity occurs and, perhaps, how it could be remedied.

Richard M. Ransohoff is at Third Rock Ventures, Boston, Massachusetts 02116, USA, and in the Department of Cell Biology,

Harvard Medical School, Boston.

e-mail:rransohoff@thirdrockventures.com

1. Wekerle, H. Acta Neurol. Scand. 136 (Suppl. 201), 22-25 (2017).

2. Jelcic, I. et al. Cell 175, 85-100 (2018).

3. Mohme, M. et al. Brain 136, 1783-1798 (2013).

4. Oksenberg, J. R., Baranzini, S. E., Sawcer, S. \& Hauser, S. L. Nature Rev. Genet. 9, 516-526 (2008).

5. Panitch, H. S., Hirsch, R. L., Haley, A. S. \& Johnson, K. P. Lancet 329, 893-895 (1987).

6. Bitsch, A., Schuchardt, J., Bunkowski, S., Kuhlmann, T. \& Bruck, W. Brain 123, 1174-1183 (2000).

7. Bruck, W. et al. Immunobiology 195, 588-600 (1996).

8. Hendriks, R. W., Yuvaraj, S. \& Kil, L. P. Nature Rev. Cancer 14, 219-232 (2014).

9. Mertin, J. Proc. R. Soc. Med. 70, 871-874 (1977).

10.Greenfield, A. L. \& Hauser, S. L. Ann. Neurol. 83, 13-26 (2018).

11. Hauser, S. L. et al. N. Engl. J. Med. 358, 676-688 (2008).

12. Wekerle, H. Autoimmunity 50, 57-60 (2017).

13. Kivisäkk, P. et al. Clin. Exp. Immunol. 129, 510-518 (2002).

14. Restorick, S. M. et al. Brain Behav. Immun. 64, 71-79 (2017).

15. Richert, J. R., McFarlin, D. E., Rose, J. W., McFarland, H. F., Greenstein, J. I. J. Neuroimmunol. 5, 317-324 (1983).

The author declares competing financial interests. See go.nature.com/2aiyh28 for details 